\title{
HeLiVa platform: integrated heart-liver-vascular systems for drug testing in human health and disease
}

\author{
Gordana Vunjak-Novakovic*1, Sangeeta Bhatia ${ }^{2}$, Christopher Chen ${ }^{3}$ and Karen Hirschi ${ }^{4}$
}

\begin{abstract}
Our project team is developing an integrated microphysiological platform with functionally connected vascular, liver and cardiac microtissues derived from a single line of human pluripotent stem cells. The platform enables functional representation of human physiology in conjunction with real-time biological readouts (via imaging and homologous reporters for all three cell phenotypes) and compatibility with high-throughput/high-content analysis. In this paper, we summarize progress made over the first year of the grant.
\end{abstract}

\section{Introduction}

The estimated cost of bringing a new drug to the clinic has increased to $\sim \$ 1.2$ billion, up from $\sim \$ 800$ million in 2003 [1]. Pharmaceutical companies face their largest losses when drugs fail late, in phase III trials and at the post-marketing stage [2]. The majority of drug recalls in the past 40 years have been due to cardiotoxicity (19\% of withdrawals, including Micturin, Fen-phen, Seldane, Vioxx, Avandia), hepatotoxicity (26\% of withdrawals, including Ananxyl, Alredase, Tasmar, Rezulin, Survector, Trovan, Cylert, Exanta, Prexige), or unpredicted adverse effects of drug interactions (for example, Posicor, Hismanal) $[3,4]$. Drug toxicities that pass through preclinical and clinical studies may result in deaths, and are highly costly. A notable example is Vioxx that has cost Merck over $\$ 5$ billion in criminal and civil settlements, and has been linked to more than 27,000 deaths [5].

Existing preclinical models can provide a critical window into human physiology for determining drug safety and efficacy. However, as many as $60 \%$ of late-stage

*Correspondence: gv2131@columbia.edu

'Columbia University, 622 West 168th Street, VC12-234, New York, NY 10032, USA Full list of author information is available at the end of the article drug development failures are due to unforeseen absorption, distribution, metabolism, excretion, and toxicity profiles that might have been predicted if models of human liver tissue were available earlier in drug development [6]. Likewise, existing heart models of cardiotoxicity do not always correlate with clinical risks. For example, preclinical hERG assays are quite helpful in identifying compounds with action potential prolongation, but are not sufficient for predicting clinical QT-proarrhythmia [4]. Human microtissues platforms are now under development to improve our capability for disease modeling $[7,8]$ and toxicology studies $[9,10]$.

Overall, the lack of predictive drug-screening systems is a critical barrier to bringing drugs to patients. In spite of major advances, existing culture systems still lack many of the structural and signaling features of native tissues, the temporal and spatial sequences of molecular and physical regulatory factors, and the dynamic forces and systemic factors provided by blood circulation. Even in the best culture settings where distinct cell types of a specific organ system are co-cultured to promote tissuespecific transport and signaling, the effects of organorgan interactions, metabolic/oxygen gradients, and cellular trafficking through tissues are still not included $[9,11]$. Also, engineered tissues have only scratched the surface in attempting to model human disease. Animal models often fail to capture human-specific features, and offer only limited control of and insight into specific mechanisms. As a result, disconnect between in vitro studies, translational animal models, and human clinical studies decreases the effectiveness of the resulting therapeutic strategies. Functional human tissue units, engineered to combine biological fidelity with the use of high-throughput platforms and real-time measurement of physiological responses, would be transformative to drug screening and predictive modeling of disease.

Modeling integrated human physiology in vitro is a formidable goal that has not been reached with any of the existing cell/tissue systems. Tissue engineering is now becoming increasingly successful in more authentically representing the actual environmental milieu of 
development, regeneration and disease progression, and in providing real-time insights into cellular and morphogenic events. While it is unreasonable to expect threedimensional tissue models to exactly match native tissues, the models can still recapitulate certain physiological functions and be used to investigate the efficacy, safety and mode of action of therapeutic agents. Instead of attempting to achieve the entire complexity of an organ, we seek to identify the simplest functional tissue unit allowing predictive in vitro studies of normal physiology and disease. Such a minimally functional tissue unit would replicate the tissue-specific architecture (as a basis for function in most tissues) and a subset of most relevant functions.

Derivation of multiple microtissues (such as heart, liver, vasculature) starting from a single population of human induced pluripotent stem (iPS) cells allows us to reach into a large genotype pool and use both the healthy cells and cells with genetic mutations for drug screening and modeling of disease. Modular microtissue platforms can then capture the salient features of multiple human tissues, by integrating tissue-specific cues within the microtissue unit (to induce physiologic cell function) with the vascular network (to assemble miniature yet functional tissue modules). These microphysiological platforms can be further interfaced with functional imaging, to enable real-time monitoring of physiological responses at molecular, cellular and tissue levels. Such interactive microtissue systems offer enormous complexity and diversity of responses to drugs and disease that can be modeled (for example, cardiotoxicity of drugs metabolized by liver in a diverse genotype pool of patients, inflammation, combinations of disease genotypes and disease environments).

We propose that human tissues with utility for microphysiological studies need to combine biological complexity (multicellular composition, normal and disease phenotypes, tissue-specific architectures, vascularization, normal human biology) and compatibility with high-throughput screening platforms (small size, easy handling, online readouts). With this in mind, we are developing integrated tissue platforms that faithfully represent the human vascular network, metabolizing liver lobule and working cardiac muscle

\section{Project goals and objectives}

In the current (UH2) phase of work, we are establishing iPS cell-based vascular, liver and cardiac microtissues providing tissue-specific architectures with an integrated vascular network, microfluidic endothelialized connections between the tissue modules, functional representation of human biology of health, injury and disease (real-time biological readouts), and compatibility with high-throughput multi-tissue platforms for studies of drug toxicity and over long periods of time ( $\geq 4$ weeks). The work is being done using iPS cells (to provide a large diversity of normal and disease genotypes) with nondestructive monitoring of the tissue architecture and function (for real-time insights into the progression of biological responses). In the subsequent (UH3) phase of work, we aim to deploy an integrated cardiac-hepaticvascular platform and demonstrate its utility for predictive studies of human physiology.

Towards these goals, we are pursuing a set of coordinated aims with constant feedback and monitoring of the milestones. Aim 1 is to develop cell-type specific labeling and sensing systems for online assessment of tissue architecture and cell function. Aim 2 is to develop a perfusable branching vascular network serving as a model of the vascular bed and for assembling vascularized liver and cardiac tissues. Aim 3 is to develop a liver module by assembling hepatic microtissues in hydrogel around the vascular tree. Aim 4 is to develop a cardiac module by assembling matured cardiac microtissues in hydrogel around the vascular tree. Aim 5 is to conduct studies of disease susceptibility, for the individual tissue modules and in the multi-tissue platform. Aim 6 is to investigate human physiology and disease in multi-tissue platforms. This way, we aim to develop a new technology for studies of drugs in human tissue models. If successful, the proposed approach would radically enhance the translation of drug discovery into human applications.

\section{Summary of current progress}

Representative results for the first project year are summarized in Figures 1 and 2, and in several publications $[8,10,12-27]$.

\section{Induced pluripotent stem reporter lines of endothelial cells, cardiomyocytes and hepatocytes}

Our laboratories have continued interest and strong expertise in biophysical regulation of the fate and function of stem cells and their differentiated progeny using molecular, cellular, matrix-derived and physical factors. We will continue to use these approaches for directed differentiation of stem cells into endothelial, cardiac and hepatic lineages, and maturation of the resulting differentiated cells.

To create clinically relevant human tissue models, we aim to generate all cell types needed for tissue construction from the same batch of iPS cells. In order to allow use of molecular and functional imaging and study physiological processes at multiple hierarchical levels and in real time, we are now incorporating biosensors (reporters) into the iPS cells to monitor specific cell phenotypes in culture (for example, distinguish between endothelial cells and cardiomyocytes), and to monitor 


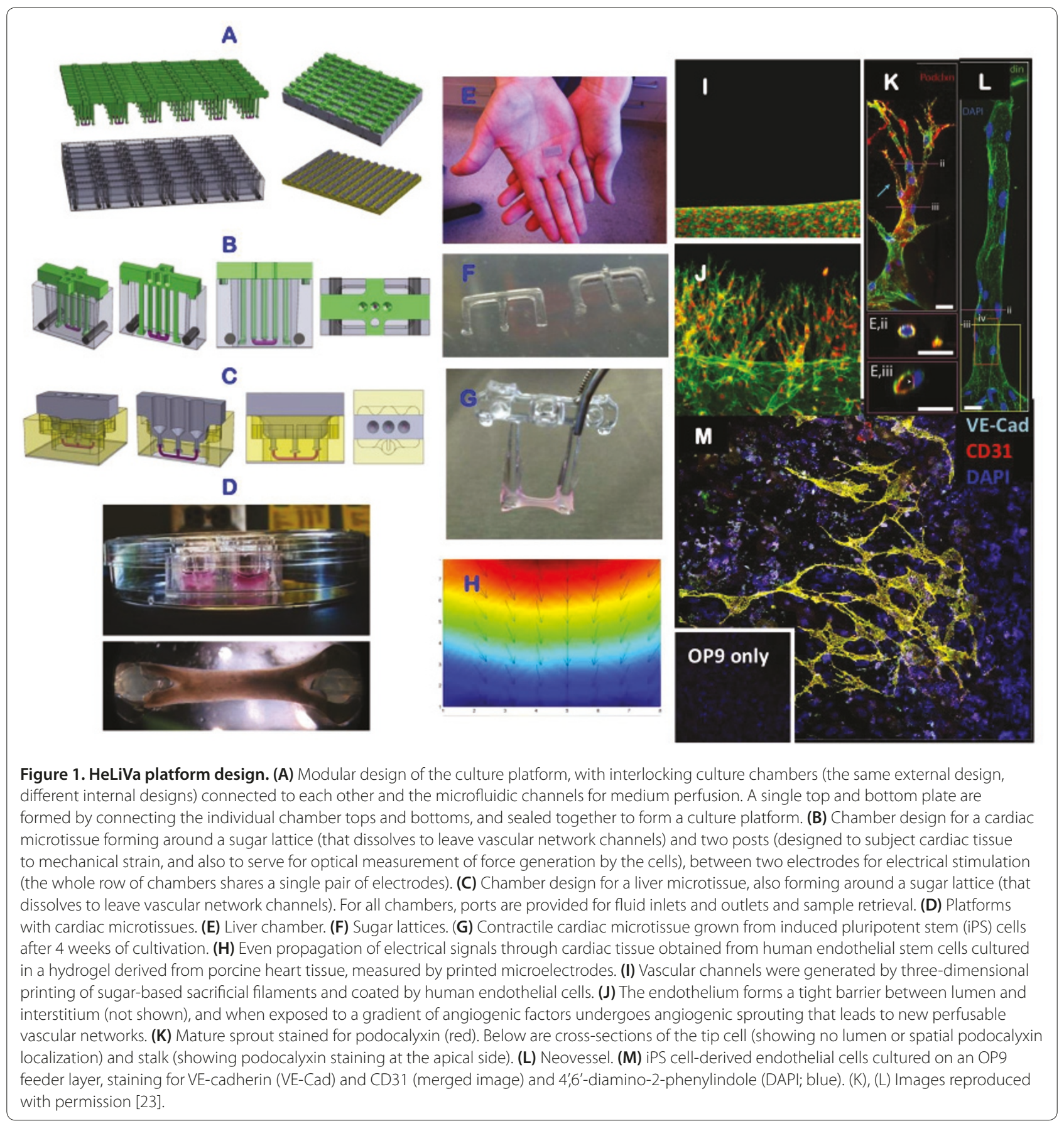

functional readouts for tissue cells. Thus far, we have established culture conditions for routine and robust generation of endothelial cells that exhibit co-expression of cell surface markers (that is, CD31, VE-cadherin) and functional properties (endothelial nitric oxide synthase production, tube formation) from multiple iPS cell lines with at least 10 to $40 \%$ efficiency [19]. We have recently adopted the culture conditions used by the Bhatia and Vunjak-Novakovic laboratories to maintain the specific iPS cell line that we are using to create the reporter lines
(iPS C2a). We are now optimizing the conditions for endothelial cell-specific differentiation of this specific cell line. We are now generating reporter cell lines using Talens [28] for homologous recombination in the iPS C2a cells (iPS endothelial cells, VEcad/GFP; iPS cardiomyocytes, beta-MHC/FRFP; and iPS hepatocytes, CYP3A4/RFP).

\section{Perfusable vascular networks}

By this time, we have developed a general approach to rapidly construct perfusable vascular networks to 


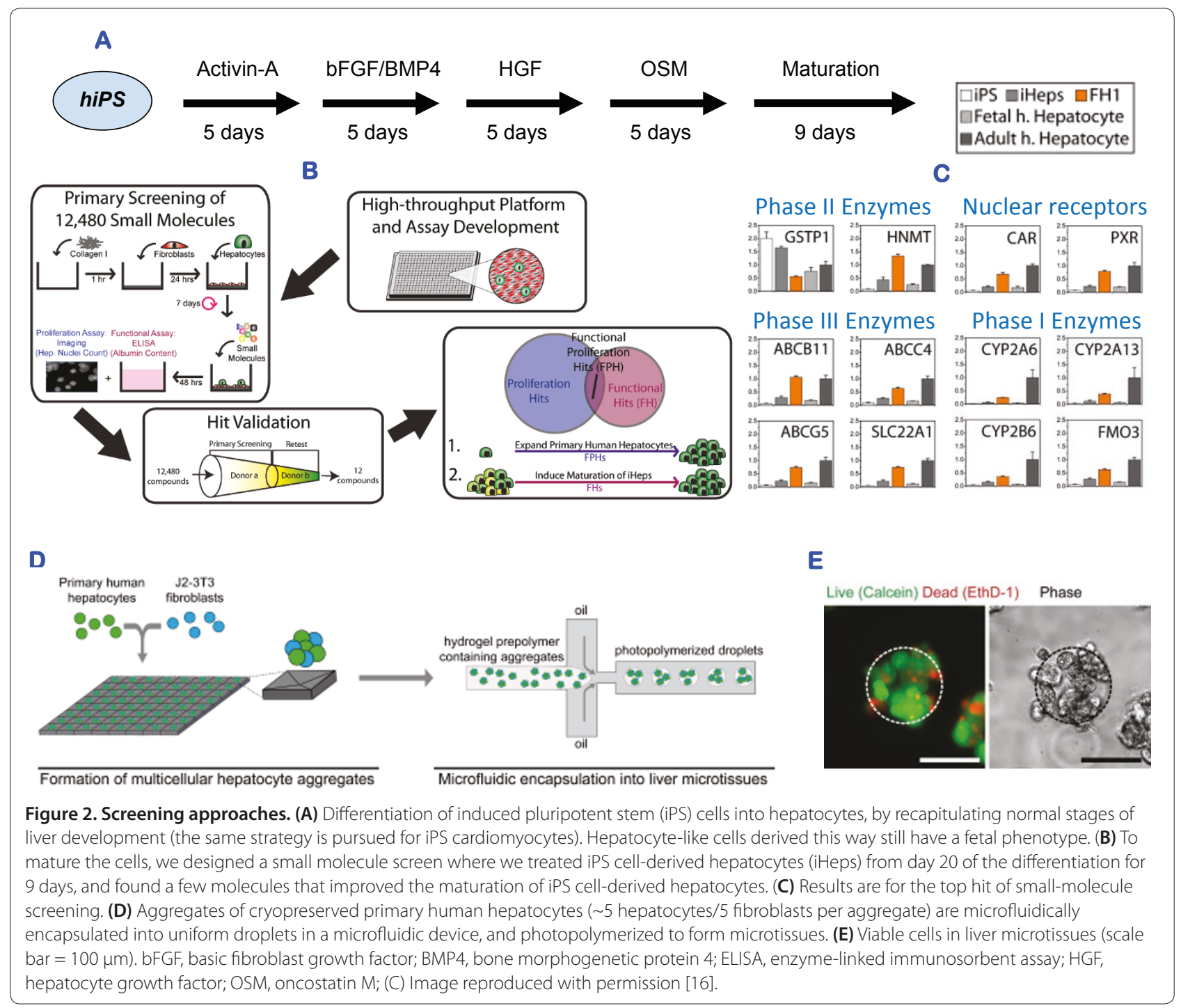

support three-dimensional culture of tissues. We printed three-dimensionally the rigid filament networks of a carbohydrate glass, and used them as a cytocompatible sacrificial template in engineered tissues containing living cells to generate networks lined with endothelial cells and perfused with blood under high-pressure pulsatile flow [20-23]. We have developed several methods to generate channel architectures within matrix scaffolds using either sacrificial filaments printed in sugars [20] or gelatin [22], or using needles that are withdrawn from the material [23]. In all of these cases, seeding human endothelial cells into these channels results in the formation of well-developed endothelium that exhibits characteristics of functional endothelium. We have observed intraluminal flows at high rates relative to extraluminal flow, and identified tight junctions that were permeable to transluminal leakage only when cells are exposed to vasoactive permeating factors.
To facilitate assembly of the HeLiVa platform, we have also developed a mechanism that allows easy plug-in of input and output microfluidic tubing to ultimately drive the perfusion within the vascular bed and connect multiple organ systems [22]. In parallel, we have begun to explore methods to integrate the vascular perfusion approaches with the cardiac microtissue platform.

\section{Liver microtissues}

We initially focused on generating hepatic microtissues from cryopreserved human hepatocytes, and then extended the established methods to a variety of cell types including iPS cell-derived hepatocytes. An integral part of this effort was the development of platforms for scalable control of tissue architectures [24,27]. To help maintain hepatocyte phenotype, we first co-cultured the primary hepatocytes with supportive fibroblasts by seeding the two cell types into an array of pyramidal 
microwells. Defined clusters of hepatocytes and fibroblasts (approximately five hepatocytes and five fibroblasts) in each microwell formed multicellular aggregates over 24 hours. These primary hepatocyte aggregates were then removed, encapsulated in microfluidically generated droplets of polyethylene glycol diacrylate prepolymer, and photopolymerized to form hepatocyte-laden hydrogel microtissues. Resulting liver microtissues are viable as evaluated by live-dead staining, and exhibit stable phenotypic function, secreting albumin for over 2 weeks. Towards an iPS cell-derived liver module, we have generated morphologically identifiable hepatocytes from human iPS cells with differentiation efficiencies $>90 \%$, as quantified by albumin and $\alpha_{1}$-antitrypsin immunofluorescence, and identified small molecules for human hepatocyte expansion and iPS cell differentiation [16]. iPS cell-derived hepatocytes were additionally validated through characterization of urea production, albumin and $\alpha_{1}$-antitrypsin secretion, and phase I drug metabolism activity. Notably, liver microtissues were successfully used to model hepatitis C infection [25].

\section{Heart microtissues}

We established protocols for differentiation of iPS cell lines into contractile cardiomyocytes, for three lines of iPS cells. Over the last 6 months we focused on one specific line, iPS $\mathrm{C} 2 \mathrm{a}$, which is now shared between all four laboratories. After systematically investigating several variations of staged molecular induction in three different settings (embryoid bodies, monolayers and pseudo-monolayers), we came to our protocol of choice that yields $\sim 65 \%$ of differentiated cardiomyocytes (as evaluated by Troponin staining and contractile function). By micromanipulation (picking of beating areas), the yield of the protocol can be increased to $>80 \%$, as compared with our target go-no-go criterion of $>30 \%$. Simple microfluidic platforms $[12,27]$ were instrumental in the development of protocols for directed differentiation of stem cells.

The resulting iPS cardiomyocytes, although functional, remain immature. To promote cardiomyocyte maturation, we applied electromechanical conditioning, following molecular induction (7 days, $5 \mathrm{~V} / \mathrm{cm}, 2 \mathrm{~ms}$ square waves, frequency $=0,0.5 \mathrm{~Hz}, 1 \mathrm{~Hz}, 2 \mathrm{~Hz}$ ). The protocols were fully established for human embryonic stem cell cardiomyocytes and are now translated to iPS C2a cardiomyocytes. Electrical stimulation increased the strain of contractions in cardiac microtissues. In parallel, the contractions became synchronized and the strain generated by cardiac microtissues increased as the stimulation frequency increased.

\section{Platform integration}

We have developed the second working prototype of our platform, in which we have tested the assembly and perfusion of cardiac microtissues formed from iPS C2a cells, and recently started testing with iPS hepatocytes. The culture chambers have a space for the formation of cardiac microtissues from a suspension of iPS cardiomyocyte micro-aggregates in hydrogel. The hydrogel forms around the sugar lattice, between two electrodes, and around two posts (that at the same time stretch the cardiac microtissues, and enable optical measurement of the force generated by the cells from the deflection of the posts). To design the posts (material composition, geometry) we conducted analyses of the strain distribution in contracting cardiac organoids. We are now assembling subsections of the integrated microphysiologic platform, for further testing and for obtaining feedback to the individual chamber design.

\section{Future directions}

We have established methods to derive, starting from the same population of iPS cells (using the line C2a as the main experimental model), functional endothelial cells, cardiomyocytes and hepatocytes of high fidelity. We have also developed methods for maturation of differentiated cells, using small molecules (for hepatocytes) and physical signals (for cardiomyocytes). The first prototypes of microfluidic platforms for the formation and cultivation of vascular, cardiac and hepatic microtissues are now being used to demonstrate utility of human iPS cellderived microtissues for physiological and pharmacological studies. Our immediate goals are to refine our microtissues by incorporating the vascular bed, to establish modular multi-tissue platforms, and to use these platforms in studies of interactive responses of cardiac, vascular and hepatic microtissues to pharmacological agents, physiological and pathological stimuli.

\section{Abbreviations \\ iPS, induced pluripotent stem. \\ Competing interests \\ The authors declare that they have no competing interests.}

\section{Acknowledgements}

The work summarized here has been planned and conducted in close collaboration of the four laboratories at Columbia University, Massachusetts Institute of Technology, University of Pennsylvania (the PI now relocated to Boston University and Wys Institute), and Yale University, within the Microphysiological Systems Initiative and with funding support from the National Institutes of Health (grant UH2 EB017103). The publication costs for this article were funded by NIH grant UH2 EB 17103

\section{Declarations}

Publication of this supplement has not been supported by sponsorship. Articles have undergone the journal's standard review process. The Editors declare that they have no competing interests.

This article has been published as part of Stem Cell Research \& Therapy Volume 4 Supplement 1, 2013: Stem cells on bioengineered microphysiological platforms for disease modeling and drug testing. The full contents of the supplement are available online at http://www.stemcellres.com/supplements/4/S1. 


\section{Author details}

'Columbia University, 622 West 168th Street, VC12-234, New York, NY 10032, USA. ${ }^{2}$ Massachusetts Institute of Technology, 77 Massachusetts Ave., Bldg 76-453, Cambridge, MA 02139, USA. ${ }^{3}$ Boston University and Wyss Institute for Biologically Inspired Engineering, Harvard University, 36 Cummington Mall, Room 201, Boston, MA 02215, USA. ${ }^{4}$ Yale University, 10 Amistad Street, POB 208089, New Haven, CT 06520, USA.

Published: 20 December 2013

\section{References}

1. DiMasi JA, Hansen RW, Grabowski HG: The price of innovation: new estimates of drug development costs. J Health Econ 2003, 22:151-185.

2. Gobaa S, Hoehnel S, Roccio M, Negro A, Kobel S, Lutolf MP: Artificial niche microarrays for probing single stem cell fate in high throughput. Nat Methods 2011, 8:949-955.

3. Recalls, Market Withdrawals, \& Safety Alerts, in Archives. US Food and Drug Administration; 2012 [http://www.fda.gov/safety/recalls/archiverecalls/ default.htm]

4. Piccini JP, Whellan DJ, Berridge BR, Finkle JK, Pettit SD, Stockbridge N, Valentin JP, Vargas HM, Krucoff MW;CSRC/HESI Writing Group: Current challenges in the evaluation of cardiac safety during drug development:Translational medicine meets the Critical Path Initiative. Am Heart J 2009, 158:317-326.

5. Graham DJ, Campen D, Hui R, Spence M, Cheetham C, Levy G, Shoor S, Ray WA: Risk of acute myocardial infarction and sudden cardiac death in patients treated with cyclo-oxygenase 2 selective and non-selective nonsteroidal anti-inflammatory drugs: nested case-control study. Lancet 2005, 365:475-481.

6. Kaplowitz N: Idiosyncratic drug hepatotoxicity. Nat Rev Drug Discov 2005, 4:489-499.

7. Huebsch N, Mooney DJ: Inspiration and application in the evolution of biomaterials. Nature 2009, 462:426-432.

8. Ploss A, Khetani SR, Jones CT, Syder AJ, Trehan K, Gaysinskaya VA, Mu K, Ritola $\mathrm{K}$, Rice CM, Bhatia SN: Persistent hepatitis C virus infection in microscale primary human hepatocyte cultures. Proc Natl Acad Sci U S A 2010, 107:3141-3145.

9. Sung JH, Kam C, Shuler ML: A microfluidic device for a pharmacokineticpharmacodynamic (PK-PD) model on a chip. Lab Chip 2010, 10:446-455.

10. Khetani SR, Bhatia SN: Microscale culture of human liver cells for drug development. Nat Biotechno/ 2008, 26:120-126

11. Gilbert P, Blau H: Engineering a stem cell house into a home. Stem Cell Res Ther 2011, 2:3.

12. Cimetta E, Sirabella D, Yeager K, Davidson K, Simon J, Moon RT, VunjakNovakovic G: Dynamic regulation of early mesodermal commitment in human pluripotent stem cells using microfluidic technologies. Lab on a Chip 2013, 13:355-364.

13. Duan Y, Liu Z, O'Neill J, Wan L, Freytes DO, Vunjak-Novakovic G: Hydrogel derived from native heart matrix induces cardiac differentiation of human embryonic stem cells without supplemental growth factors. J Cardiovasc Transl Res 2011, 4:605-615.
14. Eng GM, Lee WB, Parsa H, Chin C, Schneider J, Linkov G, Vunjak-Novakovic G: Engineering complex three-dimensional cell microenvironments using docking hydrogel shapes. Proc Natl Acad Sci U S A 2013, 110:4551-4556.

15. Kosztin I, Vunjak-Novakovic G, Forgacs G: Multiscale modeling of multicellular systems: Application to tissue engineering. Rev Mod Phys 2012, 84:1791-1805.

16. Shan J, Schwartz RE, Ross NT, Logan DJ, Thomas D, Duncan SA, North TE, Goessling W, Carpenter AE, Bhatia SN: Identification of small molecules for human hepatocyte expansion and iPS differentiation. Nat Chem Biol 2013, 9:514-520.

17. Tandon N, Marolt D, Cimetta E, Vunjak-Novakovic G: Bioreactor engineering of stem cell environments. Biotechnol Adv 2013, 31:1020-1031.

18. Zhang Y, Sevilla A, Wan LQ, Lemischka I, Vunjak-Novakovic G: Patterning pluripotency of embryonic stem cells. Stem Cells 2013, 31:1806-1815.

19. Chaudhury H, Goldie LC, Hirschi KK: Vascular precursor cells. Genes Cancer 2012, 2:1081-1084

20. Miller JS, Stevens KR, Yang MT, Baker BM, Nguyen DT, Cohen DM, Toro E, Chen SS, Galie PA, Yu X, Chaturvedi R, Bhatia SN, Chen CS: Rapid casting of patterned vascular networks for perfusable engineered threedimensional tissues. Nat Mater 2012, 11:768-774.

21. Baranski JD, Chaturvedi RR, Stevens KR, Eyckmans J, Carvalho B, Solorzano RD, Yang MT, Miller JS, Bhatia SN, Chen CS: Geometric control of vascular networks to enhance engineered tissue integration and function. Proc Natl Acad Sci U S A 2013, 110:7586-7591.

22. Baker BM, Trappmann B, Stapleton SC, Toro E, Chen CS: Microfluidics embedded within extracellular matrix to define vascular architectures and pattern diffusive gradients. Lab Chip 2013, 13:3246-3252.

23. Nguyen DHT, Stapleton SC, Yang MT, Cha SS, Choi CK, Galie PA, Chen CS: Biomimetic model to reconstitute angiogenic sprouting morphogenesis in vitro. Proc Natl Acad Sci U S A 2013, 110:6712-6717.

24. Stevens KR, Ungrin MD, Schwartz RE, Ng S, Carvalho B, Christine KS, Chaturvedi RR, Li CY, Zandstra PW, Chen CS, Bhatia SN: InVERT molding for scalable control of tissue microarchitecture. Nat Commun 2013, 4:1847.

25. Schwartz RE, Trehan K, Andrus L, Sheahan TP, Ploss A, Duncan SA, Rice CM, Bhatia SN: Modeling hepatitis $C$ virus infection using human induced pluripotent stem cells. Proc Natl Acad Sci U S A 2012, 109:2544-2548.

26. Marcelo KL, Goldie LC, Hirschi KK: Endothelial cell differentiation and specification. Circ Res 2013, 112:1272-1287.

27. Cimetta E, Godier-Furnémont A, Vunjak-Novakovic G: Bioengineering heart tissue for in vitro testing. Curr Opin Biotechnol 2013, 24:926-932.

28. Hockemeyer D, Wang H, Kiani S, Lai CS, Gao Q, Cassady JP, Cost GJ, Zhang L, Santiago Y, Miller JC, Zeitler B, Cherone JM, Meng X, Hinkley SJ, Rebar EJ, Gregory PD, Urnov FD, Jaenisch R: Genetic engineering of human pluripotent cells using TALE nucleases. Nat Biotechnol 2011, 29:731-734.

\section{doi:10.1186/scrt369}

Cite this article as: Vunjak-Novakovic G, et al: HeLiVa platform: integrated heart-liver-vascular systems for drug testing in human health and disease. Stem Cell Research \& Therapy 2013, 4(Suppl 1):S8. 